\title{
Sozialpartnerschaft bei Arbeitgeber- verbänden: „Schnee von gestern“ oder vor der Renaissance?
}

\begin{abstract}
Was bedeutet Sozialpartnerschaft für Arbeitgeberverbände und wie verbreitet ist sie? In der Diskussion um die Erosion der klassischen Tarifpolitik in Deutschland wird oft übersehen, wie sich die Arbeitgeberverbände zur Sozialpartnerschaft positionieren. Erstaunlicherweise hat in den letzten Jahren die positive Bezugnahme auf Sozialpartnerschaft bei den Arbeitgeberverbänden zugenommen. Diese Veränderung verläuft aber in sich heterogen und zeigt starke Abweichungen zwischen traditionellen und neueren Branchen. Während sich in traditionellen Branchen eine partielle Wiederbelebung sozial- und konfliktpartnerschaftlicher Orientierungen zeigt, sind Branchen neueren Typs von Orientierungen geprägt, die sich eher als brüchige Partnerschaft interpretieren lassen.
\end{abstract}

MARKUS HELFEN

\section{Einleitung}

„Sozialpartnerschaft“ ist einer der schillerndsten Begriffe der deutschen industriellen Beziehungen überhaupt (für eine Begriffsentfaltung vgl. Kädtler 2012). In vielerlei Hinsicht als zu unbestimmt und oft als ideologisch aufgeladen verpönt, erscheint dieser Begriff als Zugang zur Analyse von Arbeitsbeziehungen zunehmend ungeeignet. Allein schon seine Unschärfe macht den Begriff „Sozialpartnerschaft" aus wissenschaftlicher Sicht suspekt, findet doch allein Haipeter (2012) sechs verschiedene Definitionen: Sie reichen von der betriebspaternalistischen Vereinnahmung der Beschäftigten bis hin zu eingespielten Verhandlungsmustern der Tarifpolitik und der (wirtschafts-)politischen Makrokoordination. Und trotz ihrer diskursiven Wiederbelebung in der Krisenbewältigung der Jahre 2008/2009 wird "Sozialpartnerschaft“ in der deutschen Debatte eher unter den Vorzeichen einer mittlerweile fast zwei Jahrzehnte andauernden, schleichenden Erosion traditioneller Tarifbeziehungen betrachtet (Kädtler 2012; Ellguth/Kohaut 2012; Streeck/Hassel 2003; Artus 2001). Insbesondere steht infrage, inwieweit Sozialpartnerschaft noch als typbildendes Element angesehen werden kann. Kurz zusammengefasst scheint es geradezu, dass niemand über Sozialpartnerschaft reden möchte, solange sie besteht. Erscheint sie aber geschwächt, wird ihr „Geist“ als Leitwert beschworen (Tullius/ Wolf 2012).
Dennoch, und vor dem Hintergrund der innerdeutschen Debatte erstaunlich, heben Beobachter von Arbeitsbeziehungen im internationalen Vergleich die „Partnerschaftlichkeit“ der deutschen Arbeitsbeziehungen als systemprägendes Element hervor (bspw. Turner 1998). Gerade die kooperative Kleinarbeitung des lohnpolitischen Konflikts zwischen den sogenannten Sozialpartnern dient als eines der wesentlichen Kriterien, um Deutschland als Prototyp in die Reihe der „,koordinierten Marktwirtschaften“ einzureihen und von den liberalen Spielarten des Kapitalismus mit eher konfliktreichen Arbeitsbeziehungen abzugrenzen (Hall/Soskice 2001). Auch wird gerade in angelsächsischen Ländern mit ihren konfliktreichen Arbeitsbeziehungen darüber spekuliert, ob und wie sie von einer auf gegenseitiger Anerkennung beruhenden Partnerschaft profitieren können (Bacon/Blyton 2007; Hyman 2005).

Vor diesem Hintergrund befasst sich der vorliegende Beitrag damit, inwieweit und in welchen Formen Sozialpartnerschaft als Orientierung für heutige Arbeitgeberverbände dient. Die Analyse geht in zwei Schritten vor: $\mathrm{Zu}$ nächst wird herausgearbeitet, welche Bedeutung normative Orientierungen für die Institutionen der industriellen Beziehungen besitzen. Hierzu werden die besonderen Merkmale der Institutionalisierung des Lohnkonflikts herangezogen, um Sozialpartnerschaft als instrumentell-pragmatische Orientierung einzuordnen, deren normativer Gehalt dazu beiträgt, dem gesetzlichen Rahmen der Arbeitsbeziehungen (u. a. Tarifvertragsgesetz, 
Betriebsverfassung) ein spezifisches Gepräge zu geben. Zugleich wird aufgezeigt, inwieweit die Arbeitgeberverbände als Vermittler von Sozialpartnerschaft - als ,ideational brokers" (Campbell 2004, S. 105) - betrachtet werden können und welcher Einfluss ihre Vermittlungsleistung auf die Kontinuität der Tarifpolitik hat (Abschnitt 2).

Ferner (4) wird anhand einer vergleichenden, qualitativexplorativen Empirie ermittelt, was Vertreter ausgewählter deutscher Arbeitgeberverbände unter dem Begriff Sozialpartnerschaft verstehen. ${ }^{1}$ Ausgangspunkt dieser Betrachtung ist der überraschende Befund, dass sozialpartnerschaftliche Orientierungen zwischen 2005/06 und 2012 wieder zugenommen haben (3). Als zentrales Ergebnis stellt sich aber heraus, dass nicht von der einen Sozialpartnerschaft gesprochen werden kann, sondern dass sich die Orientierungen der Arbeitgeberverbände qualitativ nach Sektoren unterscheiden. Die Spannweite reicht dabei von einer kooperativen Form der Sozialpartnerschaft (bspw. Chemieindustrie) über die „Konfliktpartnerschaft“ (bspw. Metallindustrie) bis hin zu einer „brüchigen Partnerschaft“ (bspw. Arbeitnehmerüberlassung). In der Diskussion der Implikationen dieser Heterogenität wird deutlich, dass die Übertragung sozialpartnerschaftlicher Orientierungen von etablierten Wirtschaftssegmenten auf neuere Bereiche die künftige Entwicklung der deutschen Tarifbeziehungen entscheidend mit beeinflusst (5).

\section{Sozialpartnerschaft als normative Orientierung}

Primär verfolgen die arbeitspolitischen Kollektivakteure, Gewerkschaften und Arbeitgeberverbände, ihre ökonomischen Interessen als Arbeitsmarktparteien, die in konfliktbeladenen Interaktionen austariert werden. Der Begriff der Institution zielt in diesem Zusammenhang darauf, dass die Kollektivakteure für diese Interaktionen Erwartbarkeit benötigen, die eine geordnete Interaktion trotz widerstreitender Interessen ermöglicht, was schon Dahrendorf (1959, S. 257) veranlasst hat, von der „Institutionalisierung“ des (Klassen-)konflikts zu sprechen. Allerdings sind die Institutionen der Arbeitsbeziehungen - allen voran die der tarifpolitischen Auseinandersetzung - davon geprägt, dass die Meta-Regeln der jeweiligen Aushandlungsprozesse genauso einer Konfliktlogik unterliegen wie die einzelnen Verhandlungsgegenstände selbst. Aus dieser regelmäßigen Infragestellung durch die handelnden Parteien ergibt sich das Spannungsverhältnis von Kooperation und Konflikt, von Vertrauen und Übervorteilung, das letztlich in jeder tarifpolitischen Verhandlung praktisch anhand der zur Verfügung stehenden Machtressourcen und unter Zuhilfenahme der vorhanden Regeln neu austariert wird (Windeler/Wirth 2010).

Zur Einordnung der Sozialpartnerschaft in das Gefüge arbeitspolitischer Institutionen ist es bedeutsam, eine Un- terscheidung zwischen formaler Regelsetzung (de jure) und deren Befolgung (de facto) zu treffen. Regulative Institutionen, die gesetzlichen Grundlagen der Arbeitsbeziehungen, dienen als wirkmächtiger Referenzrahmen für die als formal angemessen geltenden Verfahren, können aber letztlich gemeinsame Problemdeutungen, Verhandlungspraktiken und Lösungen nicht in Gänze vorwegnehmen. Normative Orientierungen, die als Werturteile in Bezug auf den „richtigen“ Umgang mit diesem Spannungsverhältnis geteilt werden, können jedoch die Lücke füllen, die der mit formalen Regeln notwendig verbundene Interpretationsspielraum hinterlässt. Normative Orientierungen dienen den Gegenspielern als Orientierungspunkte, um angemessene Verhandlungspraktiken zu wählen bzw. den Praktiken des Gegenübers eine problemadäquate Bedeutung beizumessen (Cutcher-Gershenfeld/Kochan 2004). Dies geschieht darüber, dass normative Orientierungen letztlich Vertrauen stiften, dass die Gegenseite - trotz starker Anreize für ein gegenteiliges Verhalten - die getroffenen Vereinbarungen ,richtig“ einhält (Granovetter 2005). In der Tarifpolitik verzichten etwa die Parteien darauf, eine (vorübergehend) vorteilhafte Verhandlungsposition auszunutzen und ziehen Kompromissbereitschaft einer offenen Konfrontation vor (Weltz 1977).

So verstanden läuft „Sozialpartnerschaft" auf eine Orientierung deutscher Arbeitgeberverbände hinaus, die eine "kooperative Konfliktverarbeitung“ (Weltz 1977, S. 293) zwischen Kapital und Arbeit unterstützt. In Anlehnung an Campbell (2004, S. 105) können die Arbeitgeberverbände mithin als "Ideenvermittler“ angesehen werden, die eine sozialpartnerschaftliche Orientierung zwischen verschiedenen gesellschaftlichen Sphären transportieren, indem sie zwischen den Unternehmen, den Gewerkschaften und dem Staat vermitteln und auf diese Weise zu einer „Entdramatisierung " des sozialen Konfliktes beitragen (Müller-Jentsch 1999). Es geht also bei Sozialpartnerschaft primär um die Orientierung der Arbeitgeberverbände und um deren Vermittlung in den Verhandlungen zwischen den jeweiligen (Organisations-)Eliten von Verbänden und Gewerkschaften (Campbell 2004).

Als Orientierung der verbandlichen Eliten beinhaltet dies neben der bloß legalistischen Bejahung des gesetzlichen Rahmens auch eine Zustimmung zu spezifischen Ausprägungen innerhalb der von den formalen Regeln gesetzten Ordnung. Im Falle der deutschen Tarifbeziehungen bedeutet dies etwa, dass branchenweit geltende Tarifverträge rein unternehmensbezogenen Verhandlungen oder gemeinsame Sozialeinrichtungen der Gewährung sozialer Leistungen durch staatliche Instanzen vorgezogen werden. Mithin wird Sozialpartnerschaft hier als instrumentell-pragmatische Orientierung verstanden, auf deren Grundlage die

1 Die vorgestellten Befunde stammen aus dem Forschungsprojekt „Tariflosigkeit auf dem Weg zum Normalzustand?" an der Freien Universität Berlin, das von der Hans-BöcklerStiftung gefördert wird (Projektnr. 2011-466-2). 
TABELLE 1

\section{Sozialpartnerschaftsorientierung von Arbeitgeberverbänden nach Gewerkschaftsdomäne}

Angaben in Prozent und absoluten Zahlen

\begin{tabular}{|c|c|c|c|c|c|c|}
\hline \multirow{2}{*}{$\begin{array}{l}\text { Gewerkschafts- } \\
\text { domäne }\end{array}$} & \multicolumn{3}{|c|}{$2005 / 06$} & \multicolumn{3}{|c|}{2012} \\
\hline & $\begin{array}{l}\text { Ablehnung* } \\
\text { in } \%\end{array}$ & $\begin{array}{l}\text { Zustimmung } \\
\text { in } \%\end{array}$ & $\mathbf{n}$ & $\begin{array}{l}\text { Ablehnung } \\
\text { in } \%\end{array}$ & $\begin{array}{l}\text { Zustimmung } \\
\text { in } \%\end{array}$ & $\mathbf{n}$ \\
\hline & \multicolumn{6}{|c|}{ zu Statement Die deutsche Sozialpartnerschaft ist ein Vorteil im internationalen Wettbewerb: } \\
\hline IG Metall & 78,4 & 21,6 & 37 & 25,0 & 75,0 & 24 \\
\hline ver.di & 82,9 & 17,1 & 41 & 41,2 & 58,8 & 34 \\
\hline IG BCE & 47,4 & 52,6 & 19 & 18,2 & 81,8 & 11 \\
\hline IG BAU & 72,1 & 27,9 & 43 & 47,8 & 52,2 & 23 \\
\hline Sonstige & 86,7 & 13,3 & 30 & 22,7 & 77,3 & 22 \\
\hline \multirow[t]{2}{*}{ Total } & 75,9 & 24,1 & 170 & 33,3 & 66,7 & 114 \\
\hline & \multicolumn{2}{|c|}{ Pearson $\chi 2(4) 11,925^{* *}$} & \multicolumn{4}{|c|}{ Pearson $\chi 2(4) 6,111$} \\
\hline \multicolumn{7}{|c|}{ zu Statement Die Gewerkschaften sind ein verlässlicher Partner: } \\
\hline IG Metall & 89,5 & 10,5 & 38 & 37,5 & 62,5 & 24 \\
\hline ver.di & 92,7 & 7,3 & 41 & 73,5 & 26,5 & 34 \\
\hline IG BCE & 73,7 & 26,3 & 19 & 45,5 & 54,6 & 11 \\
\hline IG BAU & 65,9 & 34,1 & 44 & 78,3 & 21,7 & 23 \\
\hline Sonstige & 80,0 & 20,0 & 30 & 65,2 & 34,8 & 23 \\
\hline \multirow[t]{2}{*}{ Total } & 80,8 & 19,2 & 172 & 62,6 & 37,4 & 115 \\
\hline & \multicolumn{2}{|c|}{ Pearson $\chi 2(4) 12,50 * *$} & \multicolumn{4}{|c|}{ Pearson $\chi 2(4) 12,059 * *$} \\
\hline \multicolumn{7}{|c|}{ zu Statement Die Tarifauseinandersetzungen sind von sachlichen Argumenten geprägt: } \\
\hline IG Metall & 94,9 & 5,1 & 39 & 54,2 & 45,8 & 24 \\
\hline ver.di & 85,7 & 14,3 & 42 & 73,5 & 26,5 & 34 \\
\hline IG BCE & 73,7 & 26,3 & 19 & 54,6 & 45,5 & 11 \\
\hline IG BAU & 81,8 & 18,2 & 44 & 69,6 & 30,4 & 23 \\
\hline Sonstige & 86,7 & 13,3 & 30 & 77,3 & 22,7 & 22 \\
\hline \multirow[t]{2}{*}{ Total } & 85,6 & 14,4 & 174 & 67,5 & 32,5 & 114 \\
\hline & \multicolumn{2}{|c|}{ Pearson $\chi 2(4) 5,452$} & \multicolumn{4}{|c|}{ Pearson $\chi 2(4) 4,353$} \\
\hline
\end{tabular}

* einschließlich Unentschiedene bzw. teils/teils.

Quelle: Wirtschaftsverbände in Deutschland 2005/2006; 2012.

Arbeitgeberverbände die Gewerkschaften als gleichberechtigte Verhandlungspartner anerkennen, die getroffenen Kollektivvereinbarungen auch gegen Widerstreben in den eigenen Reihen durchsetzen und auch gemeinsam mit den Gewerkschaften Initiativen gegenüber dem Staat ergreifen (vgl. Haipeter 2012; Turner 1998).

In dynamischer Perspektive stellt Sozialpartnerschaft zwischen den Tarifparteien einen „kontinuitätssichernden Mechanismus“ (Beyer 2006, S. 263) dar, der zu einer Reproduktion und Stabilität der kooperativen Konfliktbearbeitung trotz des zugrunde liegenden Grundkonflikts beiträgt, sofern die Arbeitgeberverbände eine solche Orientierung vermitteln können. Erodieren umgekehrt sozialpartnerschaftliche Verhaltensprinzipien innerhalb der Verbände oder greifen sie nicht mehr auf neu entstehende Branchen über, wird ein institutioneller Wandel wahrscheinlich, dessen Richtung aufgrund des zugrunde liegenden Konfliktpotenzials nur schwer vorherzusehen ist (Thelen 2009).

\section{Sozialpartnerschaft der Arbeitgeber- verbände vor der Renaissance?}

Eine sozialpartnerschaftliche Orientierung ist für die Arbeitgeber erst nach dem Zweiten Weltkrieg in bewusster Abgrenzung zu den Weimarer Jahren und in Anlehnung an die katholische Soziallehre wirkmächtig geworden (exemplarisch BDA 1952; Nell-Breuning 1964; kritisch dazu Horn 1955), auch um weitergehenden Forderungen der Gewerkschaften nach Mitbestimmung und Wirtschaftsdemokratie entgegenzutreten (Müller-Jentsch 2007/2008). Diese Antinomie zwischen Mitbestimmung und Sozialpartnerschaft hat sich zwar nicht aufgelöst, sie ist jedoch mit der Zeit gegenüber einem überwiegend instrumentell-pragmatischen Umgang der Tarifparteien in den Hintergrund getreten (Behrens 2011); durchaus mit wechselseitiger rhetorischer Übernahme der Begrifflichkeiten (Kädtler 2012; 
Haipeter 2012). Heute nähren jedoch die Erosionserscheinungen der Sozialpartnerschaft in den vergangenen zwei Jahrzehnten erhebliche Zweifel an der Vermittlungsfähigkeit der Arbeitgeberverbände. Die Stichworte lauten: abnehmende (Flächen-)Tarifbindung, Verbands-Mitgliedschaften ohne Tarifbindung (OT-Mitgliedschaften), Forderung nach gesetzlichen Öffnungsklauseln, nicht-tarifgebundene Verbandsuntergliederungen, Austritte aus Arbeitgeberverbänden und fehlende Verbandsstrukturen in neuartigen Branchen (Streeck 2009; Silvia/Schroeder 2007; Haipeter/ Schilling 2006; Ettl/Heikenroth 1996).

Vor diesem Hintergrund überraschen Befunde, welche der Zustimmung zur Sozialpartnerschaft im Arbeitgeberverbandslager eine erstaunliche Renaissance bescheinigen. Im Vergleich zweier Befragungen von Geschäftsführern der Arbeitgeberverbände, und zwar einmal im Jahr 2005/2006 und einmal 2012, zeigt sich, dass sich das Meinungsbild gegenüber der Sozialpartnerschaft gedreht hat (vgl. Tabelle 1). Aktuell steht „Sozialpartnerschaft als Vorteil im internationalen Wettbewerb" bei den befragten Verbandsmanagern gegenüber dem Befragungszeitraum 2005/2006 hoch im Kurs. Während 2005/2006 eine deutliche Mehrheit dieses Statement ablehnt oder aber geteilter Meinung ist, stimmen im Jahr 2012 zwei Drittel dieser Aussage zu. Bei anderen Einschätzungen ist dieser Meinungsumschwung nicht so ausgeprägt, aber immerhin sehen jeweils rund ein Drittel der Befragten 2012 in den Gewerkschaften einen „verlässlichen Partner" und halten die Tarifauseinandersetzung für „von sachlichen Argumenten geprägt“.

Auch wenn diese Befunde insgesamt auf einen Meinungsumschwung hindeuten, bleiben die erheblichen Unterschiede in der Zustimmung zur Sozialpartnerschaft zwischen den Gewerkschaftsdomänen bestehen. Im Organisationsbereich der IG BCE ist die Zustimmung zu allen Aussagen fast durchweg am höchsten. Insbesondere im Organisationsbereich der IG Metall hat sich ein starker Umschwung ergeben, sodass die Arbeitgeberverbände heute eine deutlich positivere Einschätzung haben als noch 2005/2006. Anders stellt es sich im Organisationsbereich von ver.di dar, wo sich die Zustimmung nicht so stark erhöht hat wie im Bereich der IG Metall. Relativ betrachtet sind die Verbände im Organisationsbereich der IG BAU zurückgefallen.

Tabelle 1 kann aber nur erste Anhaltspunkte liefern, da diese Einschätzungen den Einflüssen der persönlichen Ansichten der befragten Verbandsvertreter, den Haltungen in der Verbandsmitgliedschaft oder den aktuellen Erfahrungen mit den Gewerkschaften unterliegen. Es lassen sich aber zwei Erklärungen vermuten: erstens die Krisenerfahrung der Jahre 2008/2009, in der die Gewerkschaften aufgrund eines kompromissbereiten Umgangs mit vorübergehenden Lohneinschnitten in den Augen des Widerparts Boden gegenüber den Jahren 2005/2006 gut gemacht haben (Haipeter 2012). Zweitens kann es aber auch im gesamten Zeitraum zu einer Erweiterung des Handlungsspielraums der Verbände insgesamt gekommen sein, der es ihnen ermöglicht, heutzutage Sozialpartnerschaft in einem positiveren Licht zu sehen als noch 2005/06.

\section{Sozialpartnerschaftliche Orientierungen}

Ausgehend vom Befund in Tabelle 1 wird nachfolgend anhand von qualitativen Interviews das Verständnis von Sozialpartnerschaft aufseiten der Arbeitgeber in verschiedenen Branchenkontexten explorativ untersucht (Übersicht 1). Im Zentrum der Analyse stehen die Verbandsgeschäftsführer, da sie als „key informants“ Auskunft über die Vermittlung von Sozialpartnerschaft durch Arbeitgeberverbände geben können, soweit sie im Alltagsgeschäft regelmäßig mit dem Tarifpartner verhandeln und zugleich gegenüber der Mitgliedschaft entsprechende Vereinbarungen vermitteln (vgl. Behrens/Helfen 2009). In den Interviews ging es vor allem darum, das grundsätzliche Verständnis der Befragten von Sozialpartnerschaft zu ermitteln, und zwar sowohl mit Blick auf den Verhandlungspartner als auch mit Blick auf die eigene Mitgliedschaft. Im Einzelnen stehen neben einem Interview auf der Ebene der Bundesvereinigung der Deutschen Arbeitgeberverbände (BDA) insgesamt zehn Interviews mit Repräsentanten der Arbeitgeberverbände zur Verfügung. Zusätzlich finden die Einschätzungen der jeweiligen Gewerkschaftsseite Berücksichtigung, um die Einschätzung des Gegenübers einzufangen. Die vorliegenden Daten gestatten einen explorativen Blick in die Orientierungswelt der Arbeitgeberverbände; ihre weitere Verallgemeinerung wäre durch vertiefende Untersuchungen zu prüfen.

Der Startpunkt der nachfolgenden Ergebnisdiskussion wird bei den traditionellen Branchen gelegt: Während in der Chemie- und der Bauindustrie ein positiver Bezug der Arbeitgeberverbände auf Sozialpartnerschaft erwartet wird, wird im Vergleich die Metallindustrie als Typ konflikt-

\section{ÜBERSICHT 1}

\section{Anzahl und Verteilung der Interviews}

Angaben in absoluten Zahlen

\begin{tabular}{l|c|c}
\hline & Verband & Gewerkschaft \\
\hline Metallindustrie & 3 & 2 \\
\hline Chemieindustrie & 2 & 2 \\
\hline Bauindustrie & 1 & 2 \\
\hline Industriedienstleistungen & 1 & 2 \\
\hline Leiharbeit & 3 & 3 \\
\hline Gesamt & 10 & $\mathbf{1 1}$ \\
\hline
\end{tabular}


partnerschaftlicher Orientierung diskutiert. Abschließend wendet sich die Betrachtung neuartigen Branchen zu, in denen weder Sozial- noch Konfliktpartnerschaft a priori erwartet werden können. Hier fällt die Wahl auf die industrienahen Dienstleistungen und die Leiharbeit.

\subsection{Sozialpartnerschaftlicher Typus: Chemie- und Bauindustrie}

Eine weitgehend konsensuale Form der Sozialpartnerschaft ist in der chemischen Industrie zu finden. Ausgehend von einer grundlegenden Umkehr in der Streikbereitschaft in den 1970er Jahren, haben beide Sozialpartner, also die Industriegewerkschaft Bergbau, Chemie, Energie (IG BCE) und der Bundesarbeitgeberverband Chemie e.V. (BAVC), die Geräuschlosigkeit von Tarifverhandlungen zum Markenzeichen ihrer „Chemiepartnerschaft“ (Kädtler 2006; www.chemie-sozialpartner.de) erhoben: „Durch den kontinuierlichen Aufbau und die Intensivierung des gegenseitigen Vertrauensverhältnisses hat sich zwischen IG BCE und BAVC in mehr als drei Jahrzehnten eine besondere Form der Zusammenarbeit entwickelt: Aus Konfliktparteien wurden Kooperations- und schließlich Sozialpartner [...]. Die in der chemischen Industrie heute praktizierte Sozialpartnerschaft ist die effizienteste und für die jeweiligen Mitglieder mit dem größten Nutzen verbundene Form des Umgangs zwischen den Tarifvertragsparteien“ (IG BCE/ BAVC 2009, S. 65). Streikaktivitäten sind zwar nicht gänzlich unbekannt, doch in Bezug auf die branchenweiten Tarifverhandlungen unüblich.

Wie ein ehemaliger Verhandlungsleiter einer regionalen Untergliederung des BAVC hervorhebt, geht es im Rahmen von Tarifverhandlungen in der Chemiebranche darum, ein „pragmatisches Erwartungsmanagement" zu betreiben, indem sich schon im Vorfeld der eigentlichen Verhandlungen die jeweiligen Verbandsführungen auch aus der Perspektive des Gegenübers über die Verhandlungspositionen verständigen. Die konsensuale Ausrichtung der Tarifpolitik wird durch die besondere Schlichtungsregel bestimmt, in der der BAVC und die IG BCE jeweils ihre Hauptvertreter entsenden. Entscheidungen, die aus diesem Gremium kommen, werden fast ausnahmslos umgesetzt. Diese Herangehensweise wird im Grundsatz auch von der Gewerkschaftsseite so bestätigt: „Ja, also Sozialpartnerschaft im eigentlichen Sinne bedeutet für mich, dass wir auf der [anderen] Seite, im Arbeitgeberlager, Ansprechpartner haben, mit denen ich unter fairen Bedingungen Tarifverhandlungen führen kann, [...] wo man jetzt nicht unbedingt [...] in Richtung Arbeitskampf agiert und reagiert oder kalkuliert" (Interview).

Diese Sozialpartnerschaft wird durch eine besondere Fähigkeit der Verbandsorganisationen von IG BCE und BAVC getragen, die Partikularinteressen einzuhegen, indem ein professional-technokratisches Verständnis überwiegt, Konfliktkosten vermieden und Entscheidungsrechte auf die Verbandsspitzen übertragen werden. Trotz jüngster Abweichungen aufgrund der Strukturverschiebungen in den gro- ßen Chemieunternehmen (Kädtler 2006) scheinen die Verbände an einer sozialpartnerschaftlichen Orientierung festzuhalten, die - wenn auch nicht bruchlos - qualitative Erfolge der Tarifpolitik für beide Seiten durch Koppelgeschäfte ermöglicht (bspw. Ausbildung, alternde Belegschaften).

Neben der Tarifpolitik erstreckt sich die Chemiepartnerschaft auch auf eine Vielzahl gemeinsam getragener Einrichtungen, allen voran den Chemie-Pensionsfonds (Betriebsrente), die Chemie-Stiftung Sozialpartner-Akademie (Berufs- und Weiterbildung) und den Unterstützungsverein der chemischen Industrie (soziale Notlagen, Berufseinstieg Jugendlicher). Im Jahr 2009 verständigten sich beide Seiten im sog. Wittenberg-Prozess zudem auf ein gemeinsames Sozialpartnerschafts-Dokument (IG BCE/BAVC 2009), das eine Fortentwicklung der sozialpartnerschaftlichen Orientierung auch institutionell begleitet. Hinzu kommt, dass die Chemiepartnerschaft auch dadurch eine tragfähige Orientierung für die Arbeitgeber darstellt, da sie eine Allianz für die Durchsetzung besonderer Brancheninteressen gegenüber dem Staat oder anderen gesellschaftlichen Akteuren ermöglicht. Ein Beispiel hierfür ist die gemeinsame Lobbyarbeit des Verbandes der Chemischen Industrie (VCI) und der IG Chemie Papier Keramik im Jahre 1992, welche eine Anpassung des Gentechnikgesetzes zum Ziel hatte (vgl. Kädtler/Hertle 1997, S. 260f.). Zusammengefasst kann gesagt werden, dass die „Chemiepartnerschaft“ unter den sozialpartnerschaftlichen Arrangements durch Tiefe und Breite besonders herausragt.

Auch die Bauindustrie ist für eine ausgeprägte Sozialpartnerschaft bekannt. Allerdings ist diese - anders als in der Chemieindustrie - aufgrund der Krisenerfahrungen in der jüngeren Vergangenheit deutlich in Mitleidenschaft gezogen worden. Zunächst fußt eine sozialpartnerschaftliche Orientierung beider Seiten auf Durchbrüchen in der Tarifpolitik. In den Worten eines Verbandsvertreters: „Nach dem Zweiten Weltkrieg [...] ist eine Sozialpartnerschaft gewachsen, die es geschafft hat, gemeinsame Einrichtungen ins Leben zu rufen: die Urlaubskasse, die Lohnausgleichskasse und die Zusatzversorgungskasse. [Das dokumentiert,] dass man trotz aller Streitereien doch eine gemeinsame Sache weiter getragen hat [...]. Insofern organisieren wir einen Teil des sozialen Ausgleichs, sozialen Frieden - trotz aller Konflikte. [...] Das ist im Kern die Sozialpartnerschaft." (Interview).

Auch in Bezug auf die gemeinsamen Initiativen sticht das Baugewerbe hervor. So konnte mit dem Entsendegesetz die erste branchenweite Mindestlohnregelung eingeführt werden. Ähnliche gemeinsame Initiativen sind die Verhinderung des Abbaus des „Schlechtwettergeldes“, das „SaisonKurzarbeitergeld“, aber auch gemeinsame Regelungen zu einer Ausbildungsumlage. Hinzu kommen Initiativen zum Wohnungs- und Straßenbau, die sich direkt an den Gesetzgeber richten. Ein Beispiel ist der gemeinsame Versuch der Industriegewerkschaft Bauen, Agrar, Umwelt (IG BAU) und des Zentralverbandes Deutsches Baugewerbe (ZDB), die Eigenheimzulage zu erhalten (ZDB 2004). 
Allerdings hat die krisenhafte Entwicklung am Bau ihre Spuren hinterlassen: „Wir haben natürlich schon eine Menge Veränderungen im Tarifbereich gehabt. Die Lohnentwicklung ist deutlich zurückgegangen. [...]. Die Gewerkschaft hat auf einiges verzichten müssen. Wir haben [...] den Lohnausgleich [für Feiertage bei den Sozialkassen] abgeschafft, wir haben die 40-Stunden-Woche wieder eingeführt, wir haben das zusätzliche Urlaubsgeld gekürzt, wir haben beim 13. Monatseinkommen Veränderungen vorgenommen, usw. [...] Das hat die Gewerkschaft doch geschultert, das muss man sagen, anerkennend sagen“ (Interview). Gerade in diesem Zusammenhang wird der „Sozialpartnerschaft" von Verbandsseite eine hohe Bedeutung beigemessen: „Wir musstenjaumsteuern, [...] und [Sozialpartnerschaft] ist sicherlich ein Vorteil, weil sie Stabilität bringt und Bindungswirkung und keinen politischen Streik. Und keine gesellschaftspolitischen Auseinandersetzungen über Löhne. Das ist ja alles nicht vorteilhaft, denn am Ende muss es der Staat machen und [...] daran können wir Sozialpartner eigentlich kein Interesse haben“ (Interview).

Mit der Zunahme der Untervergabe von einzelnen Bauleistungen und der Veränderung der Geschäftsmodelle der Baukonzerne in Richtung Dienstleistungen (hierzu Bosch et al. 2011) zeichnet sich bei der Gewerkschaft allerdings die Einschätzung ab, dass sich das Klima gedreht hat: „,[Die] Sozialpartnerschaft im Bau war eh immer ruppiger [...]. Eine traditionelle Sozialpartnerschaft hatten wir nie, so will ich das nicht nennen, aber sagen wir mal so, ein Geflecht aus sozialen Umgangsformen, die nicht hinten runter fallen. [...] [Heute] wird mit härteren Bandagen gefochten, das ist wirklich eindeutig. [...] Man stülpt nicht sofort alles um, [...] aber sie merken schon, dass der Wind härter wird in der Auseinandersetzung" (Interview).

\subsection{Konfliktpartnerschaftlicher Typus: Metallindustrie}

Die Beziehungen zwischen den Metallarbeitgebern und der IG Metall werden häufig als stilprägend für die deutschen Arbeitsbeziehungen insgesamt angesehen, da sie jeweils einflussreiche Segmente des Kernbereichs der deutschen Exportwirtschaft vertreten. Anders als in der Chemieindustrie und auch der Bauindustrie herrscht in der Metallindustrie aber die „Konfliktpartnerschaft“ (Müller-Jentsch 1999) vor. Diese Orientierung lässt sich am ehesten dadurch erfassen, dass in beiden Lagern jeweils sehr unterschiedliche Ansätze nach innen verfolgt werden, die dann im Zuge einer pragmatischen Aushandlung in der Tarifpolitik miteinander vermittelt werden. Im Arbeitgeberlager wird eher zurückhaltend mit dem Begriff der Sozialpartnerschaft operiert: „[...] Nach meiner Wahrnehmung ist Sozialpartnerschaft in der IG Metall ein Reizwort. Klarerweise, weil sich die IG Metall doch sehr als Gegenmacht versteht [...] und da Sozialpartnerschaft vielleicht missverstanden wird als Anbiederungskurs. Aber sicherlich gibt es auch in unseren Reihen einige, die [...] in der Auseinandersetzung ein Ziel sehen.
[Da würde] man dann ähnliche Vorbehalte zu hören bekommen [...]: , Na, ihr biedert euch bei der Gewerkschaft an'. [...] Man dürfte [Sozialpartnerschaft] [...] zumindest nicht überbetonen, um [...] nicht den Eindruck zu erwecken, man hat kampflos Dinge preisgegeben“ (Interview).

Zugleich herrscht auf Arbeitgeberseite ein Verständnis vor, das Sozialpartnerschaft mit den gesetzlichen und organisatorischen Machtressourcen auf Gewerkschaftsseite verbindet. Beispielsweise führt ein Verbandsvertreter aus: „Wenn man Sozialpartnerschaft in den Mund nimmt, dann ist das ja in der Regel das Zusammenarbeiten zwischen Arbeitgeberverbänden und Gewerkschaften. Mit dem originären Auftrag, sich um die [...] Arbeitsbedingungen [zu kümmern]: in erster Linie Tarifverträge, aber auch [...] in den jeweiligen Betrieben, wo die Gewerkschaften über die Betriebsräte und Vertrauensleute natürlich [...] auch Rechte und auch Pflichten haben, sowohl aus dem Betriebsverfassungsgesetz als auch aus dem Tarifvertragsgesetz. Das verstehe ich unter Sozialpartnerschaft" (Interview). Ein anderer betont: „Also hier in der Region ist es so, dass in den Betrieben tradiert die IG Metall vorhanden ist, und zwar mit einem hohen Organisationsgrad. Der liegt teilweise bei $80 \%$ [...] Das führt unweigerlich dazu, dass dann die Sozialpartner sehr eng zusammenarbeiten, um die Unternehmen dann auch in ihrem Bestand zu sichern [...]. Also von daher gibt es da sehr viel Gemeinsamkeiten. Im Augenblick gibt es wenige Gemeinsamkeiten, weil wir uns so kurz vor einer Tarifrunde befinden“ (Interview).

Diese konflikthafte Positionierung spiegelt sich in den Einschätzungen der IG Metall wider: „Wenn wir die Sozialpartnerschaft meinen, die darauf baut, dass man organisationsmächtig und durchsetzungsfähig ist und dann sozusagen auf gleicher Augenhöhe mit Unternehmen versucht, bestimmte Kompromisse auszuhandeln, ist das eine positive Form der Sozialpartnerschaft. Aber Sozialpartnerschaft heißt nicht, sich sozusagen der Mächtigkeit von Konzernen unterzuordnen [...]. Also Sozialpartnerschaft kann nur vernünftig zu Ergebnissen führen, wenn Gewerkschaften und wenn Arbeitnehmer auch mächtig sind“ (Interview).

Im Vergleich zur Chemie- und Bauindustrie ist nachvollziehbar, dass in Tarifauseinandersetzungen dem Streik bzw. der Streikandrohung eine größere Bedeutung zukommt. Zudem ist die Verbreitung von „OT-Verbänden“ gerade in der Metall- und Elektroindustrie ein wichtiger Konfliktherd (Haipeter/Schilling 2006). Allerdings lässt sich auch beobachten, dass die Verbände einen starken Gegner dort wertzuschätzen wissen, wo es um die verlässliche Einhaltung der gefundenen Vereinbarung geht. So etwa ein Verbandsstatement anlässlich der Krise 2008/2009: „Der von uns mitgestaltete Flächentarifvertrag und die darin enthaltenen Öffnungsklauseln ermöglichen flexible Lösungen, damit die Belastungen auf beiden Seiten vertretbar bleiben. In der aktuellen Situation wird einmal mehr deutlich, dass die funktionierende Sozialpartnerschaft nicht nur unternehmensintern viele Vorteile bringt, sondern auch einen klaren Pluspunkt im internationalen Wettbewerb darstellt." Auch 
schließt eine Konfliktpartnerschaft nicht aus, dass es zu gemeinsam getragenen Initiativen (zuletzt etwa die Abwrackprämie, vgl. IG Metall 2008; VDA 2009) und Lösungen im Bereich der Tarifpolitik kommt, die Kompromisse auf beiden Seiten erfordern. Zusammenfassend kann gesagt werden, dass die „Konfliktpartnerschaft“ in der Metallindustrie auf einem festen institutionellen Rahmen, einer relativ starken Gewerkschaftsorganisation und einem Pragmatismus auf Arbeitgeberseite beruht. Sozialpartnerschaft als Orientierung hat in diesem Gefüge ihren stabilisierenden Platz, steht aber unter einem Rechtfertigungsdruck gegenüber einer mehr oder weniger expliziten „Wir können auch anders“-Haltung.

\subsection{Brüchige „Partnerschaft“: Industriedienst- leistungen und Leiharbeit}

Mit der Branche der Industriedienstleistungen wird nun das Terrain von neuartigen Branchen betreten. Hier stellt sich heraus, dass Sozialpartnerschaft als Orientierung wohl bekannt und auch in Teilen vermittelt wird, dieser Sektor aber doch erheblichen Komplikationen unterliegt, die geeignet sind, Sozial- und auch Konfliktpartnerschaft zu unterminieren. Zunächst ist die Zahl der Akteure in diesem Feld höher und die entsprechenden Orientierungen der je beteiligten Tarifparteien sind heterogener, da bei den industriellen Dienstleistungsunternehmen verschiedene Ursprungsbranchen nachwirken: „In der Metallindustrie haben sie die IG Metall und sonst niemand: ,Es kann nur einen geben $[\ldots]$... Das ist bei uns anders: Es gibt bei uns ganz viele. Es gibt bei dem Dienstleister im Stahlwerk die IG Metall, sie finden bei dem Dienstleister auf der Raffinerie die IG BCE, sie finden bei dem Dienstleister im Bereich der Abfallwirtschaft ver.di. Also sie haben immer mit anderen Partnern zu tun [..... . Also muss ich die Sozialpartner überzeugen, dass man hier einen anderen Weg gehen muss [...] und insofern haben sie dann auch relativ schnell Tarifgemeinschaften am Tisch" (Interview). Hinzu kommt aber gerade aus Verbandsperspektive, dass es erhebliche weiße Flecken in der tarifvertraglichen Abdeckung gibt: „Natürlich kriegen sie nicht wie selbstverständlich [...] sofort alle [als] Mitgliedsfirmen [...]. Die [Unternehmen] sind natürlich teilweise mit ihren Regelungen zufrieden, wenn sie ohne Tarifvertrag was machen. Insbesondere viele Kleinere sind ja sogenannte OT-Firmen. Die suchen auch gar nicht den Tarifvertrag“" (Interview).

Aus Sicht der Gewerkschaften erfordert dies das Eingehen auf ungeliebte Kompromisskonstellationen: „Und da sagen die Gewerkschaften auch: ,Naja, bevor wir da auf der Tariflandschaftskarte einen weißen Flecken und gar keinen Tarifvertrag haben, weil die Klassischen einfach nicht anwendbar sind, machen wir einen neuen Tarifvertrag “ " (Interview). Allerdings wird auf diese Weise eine Orientierung an Sozial- und Konfliktpartnerschaft erschwert; und es entstehen Zweifel an der Orientierung der Arbeitgeberseite: „Genau das ist der Punkt und genau da versagt die ganze Branche [...]. Dass die Verantwortlichen sich zwar in Ver- bänden zusammentun, um wirtschaftliche Interessen zu bündeln, aber dass die nicht bereit sind, so einen großen Bereich in eine soziale Verantwortung zu nehmen [...] und [...] zu sagen: ,Dazu gehört auch eine vernünftige Sozialpartnerschaft, da stehen wir zu." (Interview).

Während bei den Industriedienstleistungen noch nennenswerte Reste von sozial- oder konfliktpartnerschaftlichen Orientierungen auf Arbeitgeberseite beobachtet werden können, die überwiegend aus der jeweiligen Unternehmenshistorie und den Ursprungsbranchen (Metall, Chemie, Baugewerbe) stammen, stellt sich die Situation in Bezug auf die Arbeitnehmerüberlassung besonders problematisch dar. $\mathrm{Zu}$ nächst ist festzuhalten, dass die Arbeitnehmerüberlassung außerordentlich heterogen ist, je nachdem in welchem Sektor sie eingesetzt wird. Dies gilt auch, obwohl oberflächlich betrachtet und anders als bei den Industriedienstleistungen zwischenzeitlich Mindestlohntarifverträge abgeschlossen worden sind. Verkompliziert wird die Ausbildung sozialpartnerschaftlicher Orientierungen allerdings durch den besonderen Charakter der Leiharbeit, in der einheitliche Standards immer auch dem Spannungsverhältnis ausgesetzt sind, dass unterschiedliche Vergleichsmaßstäbe je nach Kundenbranche angelegt werden. Hinzu kommt, dass sich nach der umfassenden Legalisierung der Leiharbeit im Zuge der Hartz-Reformen auf Arbeitnehmerseite eine Haltung festgesetzt hat, die der Leiharbeit nicht den Status eines legitimen Geschäftsmodells zubilligen kann (vgl. die Kampagnen www.hundertprozentich.de, 23.09.2011; www.gleichearbeitgleichesgeld.de, 23.09.2011).

Auf Arbeitgeberseite hat lange Zeit eine außerordentlich konfrontativ ausgerichtete Verbandslandschaft das ihre zu Legitimationsdefiziten und Misstrauen beigetragen. Insbesondere die langjährige Koexistenz von Tarifverträgen zwischen der Tarifgemeinschaft Christlicher Gewerkschaften für Zeitarbeit und Personal-Service-Agenturen (CGZP) und einzelnen Zeitarbeitsverbänden mit den Tarifverträgen der DGB-Gewerkschaften und dem Bundesverband Zeitarbeit (BZA) bzw. dem Interessenverband Deutscher Zeitarbeitsunternehmen (IGZ) hat das Vertrauen in die Arbeitgeberseite erschüttert (Wiedemuth/Dombre 2008). Erst die Fusionen, die überdies durch die letztlich erfolgreiche Klage der DGB-Tarifgemeinschaft gegen die Tariffähigkeit der CGZP befördert werden musste, haben zu einer einheitlicheren Tariflandschaft geführt. Die DGB-Gewerkschaften fühlen sich nach wie vor zur tarifvertraglichen Regelung einer in der Mitgliedschaft nur wenig akzeptierten Form der Arbeitserbringung gezwungen, solange sie keine Revision des Arbeitnehmerüberlassungsgesetzes erreichen können. Und auf der Arbeitgeberseite fehlt aufgrund einer unausgereiften Branchenidentität (Menez 2010) die Fähigkeit, die Vielzahl von neuen Unternehmen in der Leiharbeitsbranche wirksam zu disziplinieren, um dem Legitimationsdefizit der Arbeitnehmerüberlassung beizukommen. Diese Einschätzung wird durch wiederholte Skandale um besondere Regelverletzungen untermauert (Schröder 2009). Hinzu kommt, dass fast alle Personaldienstleistungsunterneh- 
men zugleich auch als Outsourcing-Agenturen tätig werden, was die Vertrauensbildung zusätzlich erschwert.

Vor diesem Hintergrund kann nicht überraschen, dass selbst eine „Konfliktpartnerschaft“ als Orientierung prekär ist, was sich auch in den Statements der Verbandsvertreter wiederfindet: „Nein, [Sozialpartnerschaft] ist nicht verwirklicht. Wir haben zwar einen Tarifvertrag, [...] der dadurch zustande gekommen ist, dass beide Tarifpartner damals von der Politik unter Druck gesetzt wurden [...]. Eine wirkliche Sozialpartnerschaft, wie sie in anderen Branchen normal ist, haben wir nicht“ (Interview). Diese Aussage wird getroffen, obgleich zumindest dieser Verband eine „echte Sozialpartnerschaft" anstrebt. Aus Gewerkschaftssicht ist diese Zielstellung jedoch nicht glaubwürdig: „Also diese Sozialpartnerschaft gibt es im Prinzip nicht. [...] [Die] sehe ich bei den [Verbänden] [...] nicht. Die lehnen Sozialpartnerschaft insofern ab, weil sie eigentlich Gewerkschaft ablehnen, die akzeptieren keine Betriebsräte. [...] Das wollen die alles nicht haben. Sie brauchen uns im Prinzip nur für ihre Kampagnen: ,Leiharbeit ist was Gutes.“ (Interview)

Paradoxerweise hat es aber trotz dieser Konflikte, die eine brüchige „Partnerschaft“ anzeigen, zugleich eine große Koalition zwischen den drei Arbeitgeberverbänden und den beteiligten Gewerkschaften in Bezug auf eine Eingliederung der Arbeitnehmerüberlassung in die branchenbezogene Mindestlohnregulierung sowie die Aushandlung der sogenannten Zuschlagstarifverträge mit den Einzelgewerkschaften (BAP/IGZ 2013) gegeben, was zumindest die Möglichkeit staatlicherseits unterstützter, konfliktpartnerschaftlicher Konsensbildung andeutet.

\section{Zusammenfassung und Diskussion}

„Sozialpartnerschaft“ wurde zunächst als instrumentellpragmatische Orientierung der Arbeitgeber im Rahmen gesetzlich-regulativ und organisatorisch befestigter Arbeitsbeziehungen eingeordnet. Anschließend wurde anhand von traditionellen und neuartigen Branchen aufgezeigt, inwieweit sich die hauptamtlichen Vertreter der Arbeitgeberverbände an Sozialpartnerschaft orientieren. Gemeinsam ist wohl allen Befragten, dass der Kernbereich einer sozialpartnerschaftlichen Orientierung auf dem Gebiet der Tarifpolitik gesehen wird; in variablem Ausmaß auch darüber hinaus, wenn gemeinsame sozialpolitische Einrichtungen sowie ein gemeinsames Auftreten gegenüber dem Gesetzgeber hinzukommen. Zudem ist festzustellen, dass eine sozialpartnerschaftliche Orientierung in keinem Fall mit der Ausblendung der zugrunde liegenden materiellen Interessendivergenzen einhergeht, wohl aber mit der Betonung eines konstruktiven Umgangs mit Konflikt. Aus Arbeitgebersicht stellt sich zudem heraus, dass eine Befriedigung des lohnpolitischen Konflikts sowie die Bewahrung einer vom Staat unabhängigen Lohnfindung als
Grundlinie sozialpartnerschaftlicher Orientierung angesehen werden kann.

Besonders auffällig ist, dass es erhebliche qualitative Unterschiede zwischen den einzelnen Sektoren gibt, die nicht zuletzt auch von der Orientierung der Gewerkschaften mit beeinflusst sind. Im Einzelnen lassen sich in den traditionellen Segmenten ausgewiesen konsensuale Orientierungen, etwa eine über den eigentlichen Kernbereich der Tarifverhandlungen hinausgreifende "Sozialpartnerschaft“ (allen voran die „Chemiepartnerschaft", in Teilen aber auch (noch) die Bauindustrie), von einer „Konfliktpartnerschaft“ (Metall) unterscheiden, die von einem pragmatischen Austarieren gegensätzlicher Interessenpositionen geprägt ist. Teilweise zeigen sich in den etablierten Branchen aber auch Risse und Ausnahmebereiche, die in mehr oder weniger geregelter Form aus der hauptsächlichen Orientierung ausbrechen. Dies wird insbesondere im Baugewerbe deutlich, wo die Gewerkschaftsseite angesichts teilweise dramatischer Veränderung der Wertschöpfungsstrukturen auch einen veränderten Stil der Arbeitgeberseite ausmacht. Aber selbst in der Chemieindustrie deuten sich diese Bruchlinien an, wenn mit Bezug auf die Reorganisation der großen Chemieunternehmen auf die Schwierigkeiten verwiesen wird, den Flächentarifvertrag durch eine konzessionsbereite, betriebsnahe Tarifpolitik zu stützen.

Ganz deutlich werden die Brüche mit Blick auf die neuartigen Branchen der Industriedienstleistungen und der Arbeitnehmerüberlassung, in denen sozialpartnerschaftliche Orientierungen aufseiten der Arbeitgeber zwar vorkommen, aber von den Gewerkschaften als nicht zuverlässig eingeschätzt werden. Dies liegt vor allem daran, dass eine Voraussetzung für gegenseitiges Vertrauen nicht eingehalten wird (Granovetter 2005), nämlich im Einzelnen darauf zu verzichten, einen vorübergehenden Vorteil tatsächlich auszunutzen, um eine Stabilisierung der Verhandlungsbeziehungen zu ermöglichen. Mithin erscheint die Rede von Sozialpartnerschaft (bislang) für die Arbeitgeber in diesen neuartigen Branchen als kaum gerechtfertigt, auch wenn Prozesse der Vertrauensbildung nicht ausgeschlossen sind.

Auf Grundlage dieser qualitativen Befunde lassen sich drei Punkte hervorheben, welche die Veränderungen der kollektiven Tarifbeziehungen kennzeichnen: Erstens erfährt in traditionellen Branchen, in denen sich „Sozialpartnerschaft“ oder „Konfliktpartnerschaft" über die Jahre ausgebildet haben, die Kontinuität etablierter Tarifbeziehungen durch Mechanismen der normativen Vermittlung auf Arbeitgeberverbandsseite Unterstützung, und zwar auch gegen Widerstände. Zweitens zeigt sich, dass Sozialpartnerschaft auf Arbeitgeberseite an den Randbereichen der etablierten Branchen und besonders in neuartigen Branchen nur eine sehr eingeschränkte Wirkmächtigkeit aufweist, sodass eine generelle und dauerhafte Renaissance von Sozialpartnerschaft nicht ohne Weiteres zu erwarten ist. Dies lässt sich pars pro toto an der Arbeitnehmerüberlassung und den Industriedienstleistungen veranschaulichen. Es stellt sich also die Frage, welche Richtung die Ansteckung nimmt, 
d.h. werden sozialpartnerschaftliche Orientierungen auf neuartige Segmente übertragen oder verbreitet sich das Fehlen solcher Orientierungen allmählich auch in den etablierten Bereichen? Aus Gewerkschaftssicht stellt sich heraus, dass nicht so sehr eine Pluralisierung verbandlicher Orientierungen ein Problem darstellt, als vielmehr die Beeinflussung derselben durch eigenes Handeln. Ein relevanter Punkt ist dann - wenn auch bei Weitem nicht der Einzige -, den richtigen Stil im Umgang mit dem Gegenüber zu finden, was auch ein größeres Repertoire eigener Orientierungen erfordert.

\section{LITERATUR}

Artus, I. (2001): Krise des deutschen Tarifsystems. Die Erosion des Flächentarifvertrags in Ost und West, Wiesbaden

Bacon, N./Blyton, P. (2007): Conflict for Mutual Gains?, in: Journal of Management Studies 44 (5), S. 814-834

Behrens, M. (2011): Das Paradox der Arbeitgeberverbände. Von der Schwierigkeit, durchsetzungsstarke Unternehmensinteressen kollektiv zu vertreten, Berlin

Behrens, M./Helfen, M. (2009): Innerverbandliche Heterogenität und die Vertretungswirksamkeit deutscher Arbeitgeberverbände, in: Industrielle Beziehungen 16 (1), S. 3-24

Beyer, J. (2006): Pfadabhängigkeit. Über institutionelle Kontinuität, anfällige Stabilität und fundamentalen Wandel, Frankfurt/New York

Bosch, G./Weinkopf, C./Worthmann, G. (2011): Die Fragilität des Tarifsystems. Einhaltung von Entgeltstandards und Mindestlöhnen am Beispiel des Bauhauptgewerbes, Berlin

Bundesarbeitgeberverband der Personaldienstleister/Interessenverband der Zeitarbeitsunternehmen (BAP/IGZ) (2013): Zweiter Abschluss mit ver.di über Branchenzuschläge, Presseinformation vom 22.02., Berlin/Münster

Bundesvereinigung der Deutschen Arbeitgeberverbände (BDA) (1952): Jahresbericht, 1. November 1951 bis 30. November 1952, vorgelegt der Mitgliederversammlung in Köln am 4.12., Köln

Campbell, J. L. (2004): Institutional change and globalization, Princeton

Cutcher-Gershenfeld, J./Kochan, T. (2004): Taking Stock: Collective Bargaining at the Turn of the Century, in: Industrial and Labor Relations Review 58 (1),

S. 3-26

Dahrendorf, R. (1959): Class and Class Conflict in Industrial Society, London Ellguth, P./Kohaut, S. (2012): Tarifbindung und betriebliche Interessenvertretung: Aktuelle Ergebnisse aus dem IAB-Betriebspanel 2011, in: WSI-Mitteilungen 65 (4), S. 297-305, http://www.boeckler.de/wsi_39698_39705.htm Ettl, W./Heikenroth, A. (1996): Strukturwandel, Verbandsabstinenz, Tarifflucht: Zur Lage der Unternehmen und Arbeitgeberverbände im ostdeutschen verarbeitenden Gewerbe, in: Industrielle Beziehungen 3 (2), S. 134-153

Granovetter, M. (2005): The Impact of Social Structures on Economic Outcomes, in: Journal of Economic Perspectives 19 (1), S. 33-50

Haipeter, T. (2012): Sozialpartnerschaft in und nach der Krise: Entwicklungen und Perspektiven, in: Industrielle Beziehungen 19 (4), S. 387-411

Haipeter, T./Schilling, G. (2006): Arbeitgeberverbände in der Metall- und Elektroindustrie. Tarifbindung, Organisationsentwicklung und Strategiebildung, Hamburg

Hall, P./Soskice, D. (2001): An Introduction to Varieties of Capitalism, in: Dies. (Hrsg.): Varieties of Capitalism. The Institutional Foundations of Comparative Advantage, Oxford/New York, S. 1-68

Horn, W. (1955): Oswald von Nell-Breuning S. J. contra Oswald von Nell-Breuning S. J. Der Frankfurter Gewerkschaftskongress im Spiegel der katholischen Soziallehre, in: Gewerkschaftliche Monatshefte 6 (Suppl. 1), S. 1-29

Hyman, R. (2005): Whose (social) partnership?, in: Stuart, M./Martìnez Lucio, M., (Hrsg.): Partnership and Modernization in Employment Relations, London/ New York, S. 251-265
IG Bergbau, Chemie und Energie (IG BCE)/Bundesarbeitgeberverband Chemie e.V. (BAVC) (2009): Verantwortliches Handeln in der Sozialen Marktwirtschaft - Der Wittenberg-Prozess der Chemie-Sozialpartner, Hannover/Wiesbaden

IG Metall (2008): IG Metall schlägt Programm zur Stabilisierung von Konjunktur und Beschäftigung vor, Pressemitteilung 12/11/2008, Frankfurt a. M.

Kädtler, J. (2006): Sozialpartnerschaft im Umbruch. Industrielle Beziehungen unter den Bedingungen von Globalisierung und Finanzmarktkapitalismus, Hamburg

Kädtler, J. (2012): Sozialpartnerschaft in der Krise - Bewährung oder Krise der Sozialpartnerschaft? Editorial, in: Industrielle Beziehungen 19 (4), S. 357 366

Kädtler, J./Hertle, H.-H. (1997): Sozialpartnerschaft und Industriepolitik. Strukturwandel im Organisationsbereich der IG Chemie-Papier-Keramik, Wiesbaden Menez, R. (2010): Arbeitgeber- und Wirtschaftsverbände in neuen Branchen: IKT und Zeitarbeit, in: Schroeder, W./Weßels, B. (Hrsg.): Handbuch Arbeitgeberund Wirtschaftsverbände in Deutschland, Wiesbaden, S. 183-208

Müller-Jentsch, W. (1999) (Hrsg.): Konfliktpartnerschaft. Akteure und Institutionen der industriellen Beziehungen, München/Mering

Müller-Jentsch, W. (2007/2008): Geschichte der Mitbestimmung Teil I bis III, in: Die Mitbestimmung 53 (12), S. 52-57 und 54 (1-3), S. 47-51, 48-53

Nell-Breuning, O. v. (1964): Partnerschaft, in: Beckerath, E. v. et al. (Hrsg.): Handwörterbuch der Sozialwissenschaften, Bd. 8, Stuttgart/Tübingen/Göttingen, S. 216-223

Schröder, G. (2009): Fleißig, billig, schutzlos. Leiharbeiter in Deutschland, Köln Silvia, S./Schroeder, W. (2007): Why are German employer associations declining? Arguments and Evidence, in: Comparative Political Studies 40 (12), S. $1433-1459$

Streeck, W. (2009): Re-Forming Capitalism. Institutional Change in the German Political Economy, Oxford

Streeck, W./Hassel, A. (2003): The crumbling pillars of social partnership, in: West European Politics 26 (4), S. 101-124

Thelen, K. (2009): Institutional Change in Advanced Political Economies, in: British Journal of Industrial Relations 47 (3), S. 471-498

Tullius, K./Wolf, H. (2012): Legitimationsprobleme im System industrieller Beziehungen: Krise oder Revitalisierung des sozialpartnerschaftlichen Geistes?, in: Industrielle Beziehungen 19 (4), S. 367-386

Turner, L. (1998): Fighting for Partnership. Labor and Politics in Unified Germany, Ithaca/London

Verband der Automobilindustrie (VDA) (2009): VDA begrüßt Neuregelung bei Umweltprämie, Pressemitteilung vom 03.06., Frankfurt a. M.

Weltz, F. (1977): Kooperative Konfliktverarbeitung. Ein Stil industrieller Beziehungen in deutschen Unternehmen, in: Gewerkschaftliche Monatshefte 28 (5), S. $291-301$ und 28 (8), S. 489-494

Wiedemuth, J./Dombre, R. (2008): Regulierung der Zeitarbeit durch Tarifpolitik, in: Bispinck, R. (Hrsg.): Verteilungskämpfe und Modernisierung. Aktuelle Entwicklungen in derTarifpolitik, Hamburg, S. 159-181

Windeler, A./Wirth, C. (2010): Netzwerke und Arbeit, in: Böhle, F./Voss, G. G./ Wachtler, G. (Hrsg.): Handbuch Arbeitssoziologie, Wiesbaden, S. 569-596

Zentralverband Deutsches Baugewerbe (ZDB) (2004): Abschaffung der Eigenheimzulage entspricht blankem Populismus, Pressemitteilung 10/13/2004, Berlin

\section{AUTOR}

MARKUS HELFEN, Dr., Dipl.-Volkswirt, Assistent am Management-Department, Fachbereich Wirtschaftswissenschaft der Freien Universität Berlin. Arbeitsschwerpunkte: Organisationstheorie und Personalpolitik, industrielle Beziehungen, Dienstleistungsunternehmen.

markus.helfen@fu-berlin.de 